\title{
Effect of N-acetylcysteine against Vancomycin-Induced Nephrotoxicity: A Randomized Controlled Clinical Trial
}

\author{
Shirinsadat Badri, PhD ${ }^{1,2}$; Rasool Soltani, PhD ${ }^{1 *}$; Mina Sayadi, PharmD ${ }^{3}$; Farzin Khorvash, MD ${ }^{4,5}$; Mohsen Meidani, MD4; Shahram Taheri, MD ${ }^{2}$ \\ ${ }^{1}$ Department of Clinical Pharmacy and Pharmacy Practice, Faculty of Pharmacy, Isfahan University of Medical Sciences, Isfahan, Iran \\ ${ }^{2}$ Isfahan Kidney Diseases Research Center, Isfahan University of Medical Sciences, Isfahan, Iran \\ ${ }^{3}$ Students Research Committee, Faculty of Pharmacy and Pharmaceutical Sciences, Isfahan University of Medical Sciences, Isfahan, Iran \\ ${ }^{4}$ Department of Infectious Diseases, Faculty of Medicine, Isfahan University of Medical Sciences, Isfahan, Iran \\ ${ }^{5}$ Nosocomial Infections Research Center, Isfahan University of Medical Sciences, Isfahan, Iran
}

\begin{abstract}
Background: The proposed mechanism of vancomycin-induced nephrotoxicity (VIN) is indirect production of reactive oxygen species in the kidney tissue. This study aimed to investigate the effectiveness of $\mathrm{N}$-acetylcysteine (NAC), an anti-oxidant agent, in the prevention of VIN.

Methods: Patients who received vancomycin for any indication were randomly divided to drug (NAC) and control groups. The patients in the drug group received oral NAC 600 mg every 12 hours for 10 days, starting concurrently with vancomycin. Serum creatinine ( $\mathrm{SCr}$ ) levels and blood urea nitrogen (BUN) as well as creatinine clearance $(\mathrm{CrCl})$ and 12-hour urine volume were recorded at baseline, every other day during the study, and 12 hours after the last dose of vancomycin on the 10th day. Furthermore, the cases of acute kidney injury ( $\mathrm{AKI} ; \geq 0.5 \mathrm{mg} / \mathrm{dL}$ or at least $50 \%$ increase in serum creatinine from baseline) were recorded in the two groups.

Results: Over the study period, 84 and 95 patients completed the study in drug and control groups, respectively. $\mathrm{SCr}$ and $\mathrm{CrCl}$ were significantly lower and higher, respectively, at all-time points (except for baseline) in the NAC compared to the control group. Furthermore, although not statistically significant, 12 cases of vancomycin-induced AKI were observed in the control group $(12.63 \%)$, while 4 cases $(4.76 \%)$ were reported from drug group $(P=0.066$; relative risk $[R R]=0.377,95 \% \mathrm{Cl}: 0.126-1.124)$. Conclusion: NAC has the potential for reduction of VIN. However, more studies are necessary to confirm this effect.

Keywords: Clinical trial, N-acetylcysteine, Nephrotoxicity, Reactive oxygen species, Vancomycin

Cite this article as: Badri S, Soltani R, Sayadi M, Khorvash F, Meidani M, Taheri S. Effect of N-acetylcysteine against vancomycininduced nephrotoxicity: a randomized controlled clinical trial. Arch Iran Med. 2020;23(6):397-402. doi: 10.34172/aim.2020.33.
\end{abstract}

Received: February 25, 2019, Accepted: January 21, 2020, ePublished: June 1, 2020

\section{Introduction}

Vancomycin is a glycopeptide antibiotic effective against gram-positive bacteria. It is one of the main antibiotics for the treatment of infections related to methicillin-resistant Staphylococcus aureus (MRSA). ${ }^{1,2}$ However, vancomycininduced nephrotoxicity (VIN), a known adverse effect reported in $5 \%-25 \%$ of patients as well as $35 \%$ of patients receiving concurrent aminoglycosides, ${ }^{3,4}$ has limited the clinical use of this antibiotic. On the other hand, new therapeutic guidelines have suggested higher doses (to attain trough level of $15-20 \mathrm{mg} / \mathrm{L}$ ) and longer duration of vancomycin therapy for treatment of MRSA complicated infections including bacteremia and hospital-acquired pneumonia increasing the risk of renal damage. ${ }^{5-7}$

Risk factors for vancomycin-related acute kidney injury (AKI) include increased total drug exposure (determined by area under the curve and treatment duration), preexisting renal dysfunction, and concurrent use of nephrotoxic agents. ${ }^{8}$

Although the exact mechanism of VIN is unknown, animal studies have suggested pro-inflammatory oxidation, mitochondrial dysfunction, and cellular apoptosis as the main causes of injury. ${ }^{8}$ Production of oxygen free radicals due to superoxide dismutase inhibition is a probable cause of VIN. ${ }^{9}$ In this regard, the positive effects of several antioxidant agents such as $\mathrm{N}$-acetylcysteine (NAC), ${ }^{10}$ vitamins $\mathrm{E}$ and $\mathrm{C},{ }^{10}$ curcumin, ${ }^{11}$ and atorvastatin ${ }^{12}$ have been shown in some studies in diminishing VIN.

NAC is an antioxidant with sulfhydryl donor activity contributing to the regeneration of glutathione (GSH) as a hydroxyl free radical scavenger. ${ }^{13}$ Cellular GSH level is enhanced by the action of NAC as this molecule is a precursor in the GSH synthesis pathway. ${ }^{14}$ Therefore, it can restore the impaired balance of pro-oxidants and antioxidants. So, NAC is known and used as an antioxidant agent. ${ }^{15,16}$ The preventive effect of NAC against renal toxicity due to various drugs has been shown in several animal studies. For example, it was demonstrated that renal damage induced by ifosfamide, cisplatin, and contrast agents used for angiography could be prevented 


\section{by NAC. ${ }^{17-19}$}

We found no human clinical study to investigate the effect of this antioxidant in prevention of VIN. So, this study aimed to assess the possible efficacy of NAC in the prevention of this adverse effect of vancomycin in patients receiving this antibiotic.

\section{Patients and Methods}

This was a randomized controlled clinical trial conducted in Al-Zahra hospital affiliated to Isfahan University of Medical Sciences. The participants' consent was obtained by means of a written, signed and dated informed consent form. In addition, the ethical committee of Isfahan University of Medical Sciences approved the study protocol (Project No. 294065). The study was registered in the Iranian Registry of Clinical Trials (identifier: IRCT20150721023282N3). The inclusion criteria included (1) age > 18 years; (2) receiving vancomycin for at least 10 days; (3) creatinine clearance $(\mathrm{CrCl})>60 \mathrm{~mL} / \mathrm{min}$; (4) no proteinuria and/or hematuria; (5) no renal disorder including glomerulonephritis, polycystic kidney disease, and renal artery stenosis; (6) no history of AKI; (7) no sepsis; (8) not using any other nephrotoxic drug including non-steroidal anti-inflammatory drugs, aminoglycosides, amphotericin B, cyclosporine, tacrolimus, and iodinated contrast media currently and within the last 4 days; (9) not using any other antioxidant (e.g., vitamins $\mathrm{C}$ and $\mathrm{E}$ ); and (10) no history of allergic reaction to NAC. Patients who received vancomycin (Daana, Iran) for any indication and met the inclusion criteria were randomly assigned to drug (NAC) and control groups. Simple randomization using a random numbers table allocated the patients, with even and odd numbers being assigned to drug and control groups, respectively. Allocation concealment was properly done so that the prescribing physician was unaware of the allocation sequence. Vancomycin was initially administered at $15 \mathrm{mg} / \mathrm{kg}$ IV every 12 hours followed by dose adjustment to obtain a trough concentration of $10-15 \mathrm{mg} / \mathrm{L}$. Blood samples were collected just 30 minutes before the 4 th dose of vancomycin. For each case, $5 \mathrm{~mL}$ of whole blood was taken and rapidly transferred to the hospital laboratory in a heparinized tube. After centrifugation at $5000 \mathrm{rpm}$ for 5 minutes, the serum (supernatant) was separated by sampler and used for assay. The drug concentration was determined using fluorescence polarization technique by COBAS INTEGRA 400 plus analyzer (Roche Diagnostics International Ltd, Basle, Switzerland). The patients in the drug group received oral NAC (in the form of effervescent tablets; Zambon, Switzerland) $600 \mathrm{mg}$ every 12 hours for 10 days, starting concurrently with vancomycin, while the patients in the control group received vancomycin alone. The patients were excluded from the study if vancomycin was discontinued earlier than 10 days due to any reason except for AKI (e.g., thrombocytopenia and hypersensitivity reactions). Serum creatinine (SCr) levels and blood urea nitrogen (BUN) as well as $\mathrm{CrCl}$ and 12-hour urine volume were recorded before the start of interventions, every other day during the study, and 12 hours after the last dose of vancomycin on the 10th day of therapy for all patients. SCr and BUN assays were performed using calorimetric detection kits (Pars Azmoon, Iran) after obtaining $5 \mathrm{~mL}$ of venous blood from each patient, which underwent centrifugation at $2200 \mathrm{rpm}$ for 15 minutes (Kubota, Japan). $\mathrm{CrCl}$ was calculated using the Cockcroft-Gault equation. Urine was collected in 2000-mL urine bags (Soha, Iran). The primary outcome measures were changes in SCr and BUN concentrations, urine output, and $\mathrm{CrCl}$ from the study enrollment to the end of follow-up (day 10) as well as differences in these parameters between the NAC and control groups at the evaluated points. Furthermore, the cases of AKI (defined as $\geq 0.5 \mathrm{mg} / \mathrm{dL}$ or at least $50 \%$ increase in serum creatinine from baseline) were recorded and compared in the two groups.

The sample size was calculated using the following formula ${ }^{20}$.

$\mathrm{n}=\left(\mathrm{Z}_{\alpha / 2}+\mathrm{Z}_{\beta}\right)^{2} \times\left(\sigma_{1}^{2}+\sigma_{1}^{2}\right) / \mathrm{d}^{2}$

where $n$ is sample size (for each group), $Z_{\alpha / 2}$ is the value of error $\alpha(1.96[5 \%]), Z_{\beta}$ is the value of error $\beta,(0.84$ $[20 \%]), d$ is the minimum difference between the mean values, and $\sigma_{1}$ and $\sigma_{2}$ are standard deviation of the variable in each group. Due to the lack of a similar clinical study on vancomycin nephrotoxicity, we used the values of $\mathrm{CrCl}$ (eGFR, as the main variable of the present study) from a study on cisplatin-induced nephrotoxicity ${ }^{21}$; thus, the values of $\mathrm{d}, \sigma_{1}$, and $\sigma_{2}$ were estimated at 14.3, 16.7, and 18.3 , respectively. Therefore, a sample size of at least 23 patients was obtained for each group.

For statistical analysis of the obtained data, SPSS software (SPSS Inc., USA) version 20.0 was used. The mean values of the measured variables were finally compared between the two groups. Data distribution pattern (normal vs. nonnormal) was assessed by Kolmogorov-Smirnov test. Due to non-normality of data distribution, non-parametric statistical tests were used to enhance the precision of analysis. Data were presented as mean \pm SD. Changes in the parameters within groups (at the five every-other-day assessment points) were analyzed using Friedman test. Mann-Whitney $U$ test was used to compare the values between the groups at each point. The number of AKI cases in the two groups was compared by Pearson chisquare test. For comparison of serum BUN between the groups, analysis of covariance (ANCOVA) was done with the control of baseline serum BUN as a covariate. $P<0.05$ was considered statistically significant.

\section{Results}

During the study, 266 patients were evaluated regarding 


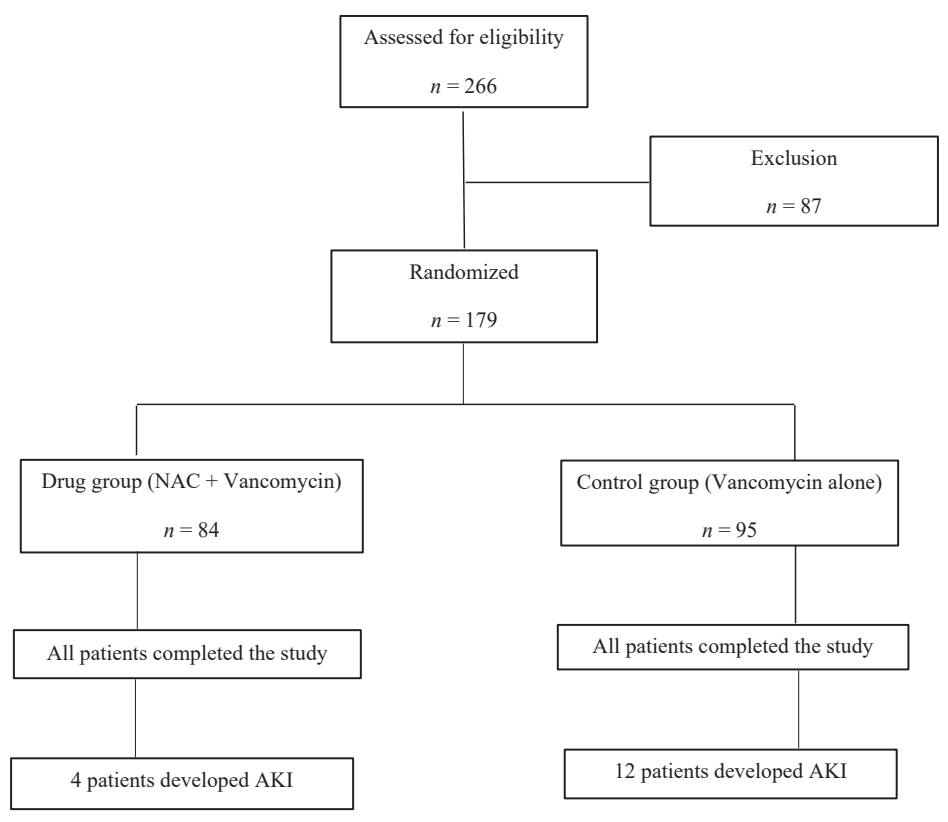

Figure 1. Patients' allocation and participation diagram. NAC, N-acetylcysteine; AKI, acute kidney injury; NAC, N-acetylcysteine.

eligibility, of whom 179 patients were eligible who were randomly assigned to the study groups (Figure 1). Finally, 84 and 95 patients completed the study in drug and control groups, respectively.

The baseline demographic and clinical properties of study patients are presented in Table 1 . As shown, both groups were matched regarding baseline values for all parameters except for BUN.

Table 2 compares the results of the evaluated parameters in the two groups over the study period. As seen, administration of NAC with vancomycin significantly prevented the increase in $\mathrm{SCr}$ and decrease in $\mathrm{CrCl}$ compared to the control group, since a significant increase in $\mathrm{SCr}$ and decrease in $\mathrm{CrCl}$ were observed in the control group. Due to the significant difference in baseline BUN between the groups, ANCOVA test was performed for this variable with the baseline values as the covariate to control any confounding effect. As shown, according to this test, NAC significantly prevented the increase in serum BUN

Table 1. Baseline Demographic and Clinical Characteristics of Study Patients

\begin{tabular}{|c|c|c|c|}
\hline \multirow[b]{2}{*}{ Parameter } & \multicolumn{2}{|c|}{ Group } & \multirow[b]{2}{*}{$P$ Value } \\
\hline & $\begin{array}{l}\text { Control } \\
(\mathrm{n}=95)\end{array}$ & $\begin{array}{c}\text { NAC } \\
(n=84)\end{array}$ & \\
\hline \multicolumn{4}{|l|}{ Gender } \\
\hline Male (n) & 61 & 67 & 0.727 \\
\hline Female $(n)$ & 23 & 28 & \\
\hline Age $(y)$ & $46.6 \pm 17.1$ & $49.2 \pm 18.7$ & 0.999 \\
\hline $\mathrm{SCr}(\mathrm{mg} / \mathrm{dL})$ & $0.93 \pm 0.16$ & $0.96 \pm 0.21$ & 0.520 \\
\hline $\mathrm{BUN}(\mathrm{mg} / \mathrm{dL})$ & $13.6 \pm 5.9$ & $17.2 \pm 7.4$ & $<0.001$ \\
\hline $\mathrm{CrCl}(\mathrm{mL} / \mathrm{min})$ & $91.6 \pm 20.8$ & $88.7 \pm 25.8$ & 0.109 \\
\hline 12-hour urine output (mL) & $1494.7 \pm 224.5$ & $1423.4 \pm 231.4$ & 0.357 \\
\hline
\end{tabular}

NAC, N-acetylcysteine; SCr, serum creatinine; BUN, blood urea nitrogen; $\mathrm{CrCl}$, creatinine clearance. compared to the control group. Furthermore, NAC had no significant effect on 12-hour urine output in comparison with the control group.

Regarding the rate of AKI in the two groups, although not statistically significant, 12 cases of vancomycininduced AKI were observed in the control group (12.63 $\%)$, while 4 cases $(4.76 \%)$ were observed in the drug group $(P=0.066$; relative risk $[\mathrm{RR}]=0.377,95 \% \mathrm{CI}$ : $0.126-1.124)$.

\section{Discussion}

In the present study, NAC could prevent the VIN shown by prevention of increase in serum creatinine and BUN as well as inhibition of reduction in $\mathrm{CrCl}$. Based on our search of the literature, most studies on prevention of VIN have used animal models. In a study by Celik et al, three different antioxidants (alpha-lipoic acid, Ginkgo biloba extract, and melatonin) and amrinone were evaluated for possible preventive effects against VIN in rats. Coadministration of all four agents with vancomycin had preventive effect against the increase of urea, creatinine and kidney malondialdehyde levels. Also, it enhanced the activities of renal superoxide dismutase and GSH peroxidase. Furthermore, these agents improved renal pathology.22 In another animal study, the protective role of atorvastatin against VIN was shown. The authors attributed this effect of atorvastatin to antagonizing renal oxidative stress associated with vancomycin use. ${ }^{12}$ In the study by Ahmida in rats, the administration of curcumin with vancomycin resulted in the reduction of the serum and renal levels of thiobarbituric acid reactive substances as well as serum urea and creatinine. It also inhibited the decrease in the activities of antioxidant enzymes and 
Table 2. Laboratory and Clinical Characteristics of Patients in Control and Drug Groups Over the Study Period

\begin{tabular}{|c|c|c|c|c|c|}
\hline \multirow{2}{*}{ Parameter } & \multirow{2}{*}{ Time } & \multicolumn{2}{|l|}{ Group } & \multirow{2}{*}{ Difference $(95 \% \mathrm{Cl})$} & \multirow{2}{*}{$P$ Value $^{\mathrm{a}}$} \\
\hline & & Control $(n=95)$ & NAC $(n=84)$ & & \\
\hline \multirow{7}{*}{$\mathrm{SCr}(\mathrm{mg} / \mathrm{dL})$} & 0 & $0.93 \pm 0.16$ & $0.96 \pm 0.21$ & $-0.030 \pm 0.028(-0.085$ to 0.025$)$ & 0.520 \\
\hline & 1 & $0.97 \pm 0.20$ & $0.89 \pm 0.19$ & $0.080 \pm 0.029(0.022$ to 0.138$)$ & 0.019 \\
\hline & 2 & $0.99 \pm 0.26$ & $0.87 \pm 0.20$ & $0.120 \pm 0.035(0.051$ to 0.190$)$ & 0.003 \\
\hline & 3 & $1.01 \pm 0.37$ & $0.85 \pm 0.23$ & $0.160 \pm 0.047(0.068$ to 0.252$)$ & 0.002 \\
\hline & 4 & $1.04 \pm 0.46$ & $0.84 \pm 0.24$ & $0.200 \pm 0.056(0.090$ to 0.310$)$ & 0.006 \\
\hline & 5 & $1.09 \pm 0.50$ & $0.89 \pm 0.30$ & $0.200 \pm 0.063(0.076$ to 0.324$)$ & $<0.001$ \\
\hline & $P$ value ${ }^{b}$ & $<0.001$ & $<0.001$ & & \\
\hline \multirow{7}{*}{$B \cup N^{c}(m g / d L)$} & 0 & $13.6 \pm 5.9$ & $17.2 \pm 7.4$ & $-3.600 \pm 0.995(-5.564$ to -1.636$)$ & $<0.001$ \\
\hline & 1 & $14.7 \pm 6.2$ & $15.7 \pm 6.9$ & $-1.000 \pm 0.979(-2.932$ to 0.932$)$ & 0.412 \\
\hline & 2 & $15.0 \pm 7.3$ & $14.8 \pm 7.8$ & $0.200 \pm 1.129(-2.028$ to 2.428$)$ & 0.529 \\
\hline & 3 & $15.7 \pm 8.8$ & $13.9 \pm 7.2$ & $1.800 \pm 1.212(-0.591$ to 4.191$)$ & 0.059 \\
\hline & 4 & $15.5 \pm 9.9$ & $14.1 \pm 7.8$ & $1.400 \pm 1.344(-1.253$ to 4.053$)$ & 0.251 \\
\hline & 5 & $16.7 \pm 11.3$ & $14.2 \pm 9.1$ & $2.500 \pm 1.547(-0.552$ to 5.552$)$ & $<0.001$ \\
\hline & $P$ value ${ }^{b}$ & $<0.001$ & $<0.001$ & & \\
\hline \multirow{7}{*}{$\mathrm{CrCl}(\mathrm{mL} / \mathrm{min})$} & 0 & $91.6 \pm 20.8$ & $88.7 \pm 25.8$ & $2.900 \pm 3.486(-3.980$ to 9.780$)$ & 0.109 \\
\hline & 1 & $87.7 \pm 20.3$ & $95.3 \pm 25.9$ & $-7.600 \pm 3.459(-14.426$ to -0.774$)$ & 0.115 \\
\hline & 2 & $87.1 \pm 25.4$ & $95.7 \pm 25.2$ & $-8.600 \pm 3.790(-16.080$ to -1.120$)$ & 0.020 \\
\hline & 3 & $87.4 \pm 26.3$ & $96.9 \pm 26.2$ & $-9.500 \pm 3.932(-17.260$ to -1.741$)$ & 0.033 \\
\hline & 4 & $87.4 \pm 27.8$ & $97.7 \pm 28.7$ & $-10.300 \pm 4.227(-18.643$ to -1.958$)$ & 0.026 \\
\hline & 5 & $82.6 \pm 25.5$ & $99.6 \pm 29.3$ & $-17.000 \pm 4.096(-25.083$ to -8.917$)$ & $<0.001$ \\
\hline & $P$ value ${ }^{b}$ & $<0.001$ & $<0.001$ & & \\
\hline \multirow{7}{*}{ Urine output $(\mathrm{mL})$} & 0 & $1494.7 \pm 224.5$ & $1423.4 \pm 231.4$ & $70.600 \pm 34.112(3.282$ to 137.918$)$ & 0.357 \\
\hline & 1 & $1481.2 \pm 342.1$ & $1391.2 \pm 312.5$ & $90.000 \pm 49.207$ (-7.108 to 187.108$)$ & 0.224 \\
\hline & 2 & $1362.8 \pm 320.1$ & $1237.8 \pm 441.6$ & $125.000 \pm 57.200$ (12.118 to 237.882$)$ & 0.450 \\
\hline & 3 & $1255.3 \pm 247.8$ & $1561.2 \pm 217.5$ & $-305.900 \pm 35.058(-375.086$ to -236.714$)$ & 0.045 \\
\hline & 4 & $1620.6 \pm 182.9$ & $1740.4 \pm 184.6$ & $-119.800 \pm 27.513(-174.095$ to -65.505$)$ & 0.790 \\
\hline & 5 & $1722.2 \pm 421.6$ & $1733.4 \pm 318.4$ & $-11.200 \pm 56.425(-122.552$ to 100.152$)$ & 0.336 \\
\hline & $P$ value ${ }^{\mathrm{b}}$ & 0.122 & 0.083 & & \\
\hline
\end{tabular}

$\mathrm{NAC}, \mathrm{N}$-acetylcysteine; $\mathrm{SCr}$, serum creatinine; BUN, blood urea nitrogen; $\mathrm{CrCl}$, creatinine clearance, $\mathrm{Cl}$, confidence interval.

${ }^{a}$ Between-group comparison (Mann-Whitney $U$ test).

${ }^{\mathrm{b}}$ Within-group comparison (Friedman test).

cANCOVA test was performed for comparison of BUN between the groups.

GSH. ${ }^{11}$ According to the results of these studies, it seems that the positive effects of NAC in our study could be due to its antioxidant effects. Vancomycin induces oxidative phosphorylation resulting in the induction of free oxygen radicals and subsequent reduction of the activity of defensive antioxidative enzymes. In animal models, administration of antioxidants expressing superoxide dismutase prevented renal tubular damage and improved kidney histology. ${ }^{23,24} \mathrm{NAC}$, with an unbound thiol group $(-\mathrm{SH})$ in its chemical structure, interacts with free radicals and enhances the production of GSH peroxidase, the main endogenous antioxidant enzyme. ${ }^{25}$ Furthermore, enhancement of superoxide dismutase activity by NAC has been shown in several studies. ${ }^{26-28}$

In the present study, there was no significant change in urine output in either the drug or the control group. Therefore, it seems that VIN does not affect this parameter and, at least in early stages, it could not be used as a marker of vancomycin-induced renal damage. However, we did not find any report on the effect of vancomycin on urine output and any judgment on this issue needs more studies. Furthermore, although the number of cases of AKI was not significantly different between the groups, as the rate of AKI was three-folds higher in the control group (12 vs 4 cases in NAC group), it is possible that use of larger sample size could yield a more significant effect.

In the current study, we used $\mathrm{SCr}$ as the main marker of VIN. However, due to limitations in detecting kidney damage using serum creatinine, alternative diagnostic measures have been proposed. SCr may remain unchanged despite a substantial renal damage and its rise may lag by about 48-72 hours after kidney injury. ${ }^{29}$ Considering that the probable mechanism of VIN is proximal tubular dysfunction, biomarkers differentiating impairment of glomerular filtration from that of tubular function including kidney injury molecule 1 and neutrophil gelatinase-associated lipocalin may improve the precision of diagnosis. ${ }^{8,30}$ Therefore, more studies using these 
biomarkers are recommended to confirm the observed desirable effects of NAC on VIN in the present study.

The main limitations of our study were the method of randomization that resulted in imbalance of the sample size between the study groups and lack of placebo group and blinding.

In conclusion, NAC has the potential for reduction of VIN. However, more studies with larger sample size are necessary to confirm this effect.

\section{Authors' Contribution}

SB and RS: developed the research idea and study design, performed data analysis and drafted the manuscript. FK, MM, and ST: selected the patients. MS: performed data gathering and patients' follow-up. All authors contributed in manuscript revision.

\section{Conflict of Interest Disclosures}

None declared.

\section{Ethical Statement}

The participants' consent was obtained by means of a written, signed and dated informed consent form. In addition, the ethical committee of Isfahan University of Medical Sciences approved the study protocol (Project No. 294065).

\section{Acknowledgements}

We acknowledge theVice-Chancellery for Research and Technology at Isfahan University of Medical Sciences that supported this research financially. In addition, we thank the personnel of Laboratory Department of Al-Zahra hospital for their assistance.

\section{References}

1. Rybak M, Lomaestro B, Rotschafer JC, Moellering R Jr, Craig $\mathrm{W}$, Billeter $\mathrm{M}$, et al. Therapeutic monitoring of vancomycin in adult patients: a consensus review of the American Society of Health System Pharmacists, the Infectious Diseases Society of America, and the Society of Infectious Diseases Pharmacists, the Infectious Diseases Pharmacists. Am J Health Syst Pharm. 2009;66(1):82-98. doi: 10.2146/ajhp080434.

2. McDougall C. Protein synthesis inhibitors and miscellaneous antibacterial agents. In: Brunton LL, Hilal-Dandan R, Knollmann BC, eds. Goodman \& Gilman's the Pharmacological Basis of Therapeutics. 13th ed. New York: McGraw Hill; 2018:1059.

3. Cunha BA. Vancomycin. Med Clin North Am. 1995;79(4):81731. doi: 10.1016/s0025-7125(16)30041-4.

4. Iwamoto T, Kagawa Y, Kojima M. Clinical efficacy of therapeutic drug monitoring in patients receiving vancomycin. Biol Pharm Bull. 2003;26(6):876-9. doi: 10.1248/bpb.26.876.

5. Elbarbry F. Vancomycin dosing and monitoring: critical evaluation of the current practice. Eur J Drug Metab Pharmacokinet. 2018;43(3):259-68. doi: 10.1007/s13318017-0456-4.

6. Rybak MJ, Lomaestro BM, Rotschafer JC, Moellering RC, Craig WA, Billeter $M$, et al. Vancomycin therapeutic guidelines: a summary of consensus recommendations from the infectious diseases Society of America, the American Society of Health-System Pharmacists and the Society of Infectious Diseases Pharmacists. Clin Infect Dis. 2009;49(3):325-7. doi: 10.1086/600877.

7. Pauly DJ, Musa DM, Lestico MR, Lindstrom MJ, Hetsko $\mathrm{CM}$. Risk of nephrotoxicity with combination vancomycinaminoglycoside antibiotic therapy. Pharmacotherapy. 1990;10(6):378-82.

8. Bamgbola O. Review of vancomycin-induced renal toxicity: an update. Ther Adv Endocrinol Metab. 2016;7(3):136-47. doi: $10.1177 / 2042018816638223$.
9. Parlakpinar H, Ozer MK, Sahna E, Vardi N, Cigremis Y, Acet A. Amikacin-induced acute renal injury in rats: protective role of melatonin. J Pineal Res. 2003;35(2):85-90. doi: 10.1034/j.1600-079x.2003.00059.x.

10. OcakS, Gorur S, Hakverdi S, Celik S, Erdogen S. Protective effects of caffeic acid phenethyl ester, vitamin C, Vitamin E and $\mathrm{N}$-acetylcysteine on vancomycin-induced nephrotoxicity in rats. Basic Clin Pharmacol Toxicol. 2007;100(5):328-33. doi: 10.1111/j.1742-7843.2007.00051.x

11. Ahmida MH. Protective role of curcumin in nephrotoxic oxidative damage induced by vancomycin in rats. Exp Toxicol Pathol. 2012;64(3):149-53. doi: 10.1016/j.etp.2010.07.010.

12. Panonnummal R, Varkey J, Dinoop DR. Protective effect of atorvastatin against vancomycin induced nephrotoxicity in albino rats. Pharmacie Globale. 2011;8(10):1-6.

13. Yalcin S, Bilgili A, Onbasilar I, Eraslan G, Ozdemir M. Synergistic action of sodium selenite and $\mathrm{N}$-acetylcysteine in acetaminophen induced liver damage. Hum Exp Toxicol. 2008;27(5):425-9. doi: 10.1177/0960327108094612.

14. Campos R, Shimizu MH, Volpini RA, de Bragança AC, Andrade $\mathrm{L}$, Lopes $\mathrm{FD}$, et al. $\mathrm{N}$-acetylcysteine prevents pulmonary edema and acute kidney injury in rats with sepsis submitted to mechanical ventilation. Am J Physiol Lung Cell Mol Physiol. 2012;302(7):L640-50. doi: 10.1152/ajplung.00097.2011.

15. Srivastava RK, Rahman Q, Kashyap MP, Lohani M, Pant AB. Ameliorative effects of dimetylthiourea and $\mathrm{N}$-acetylcysteine on nanoparticles induced cytogenotoxicity in human lung cancer cells-A549. PLoS One. 2011;6(9):e25767. doi: 10.1371/journal.pone.0025767.

16. El-SisiAel-D, El-Syaad ME, El-Desoky KI,Moussa EA. Protective effects of alpha lipoic acid versus $\mathrm{N}$-acetylcysteine on ifosfamide-induced nephrotoxicity. Toxicol Ind Health. 2015;31(2):97-107. doi: 10.1177/0748233712469649.

17. Abdel-Wahab WM, Moussa FI, Saad NA. Synergistic protective effect of $\mathrm{N}$-acetylcysteine and taurine against cisplatin-induced nephrotoxicity in rats. Drug Des Devel Ther. 2017;11:901-8. doi: 10.2147/DDDT.S131316.

18. Chen N, Aleksa K, Woodland C, Rieder M, Koren G. $\mathrm{N}$-Acetylcysteine prevents ifosfamide-induced nephrotoxicity in rats. Br J Pharmacol. 2008;153(7):1364-72. doi: 10.1038/ bjp.2008.15.

19. Kay J, Chow WH, Chan TM, Lo SK, Kwok OH, Yip A, et al. Acetylcysteine for prevention of acute deterioration of renal function following elective coronary angiography and intervention: a randomized controlled trial. JAMA. 2003;289(5):553-8. doi: 10.1001/jama.289.5.553.

20. Miot HA. Sample size in clinical and experimental trials. J Vasc Bras. 2011;10(4):275-8.

21. Yamamoto $\mathrm{Y}$, Watanabe K, Tsukiyama I, Matsushita H, Yabushita $H$, Matsuura $K$, et al. Nephroprotective effects of hydration with magnesium in patients with cervical cancer receiving cisplatin. Anticancer Res. 2015;35(4):2199-204.

22. Celik I, Cihangiroglu M, Ilhan N, Akpolat N, Akbulut HH. Protective effects of different antioxidants and amrinone on vancomycin-induced nephrotoxicity. Basic Clin Pharmacol Toxicol. 2005;97(5):325-32. doi: 10.1111/j.1742-7843.2005. pto_153.x.

23. Oktem F, Arslan M, Ozguner F, Candir O, Yilmaz H, Ciris $M$, et al.. In vivo evidences suggesting the role of oxidative stress in pathogenesis of vancomycin-induced nephrotoxicity: protection by erdosteine. Toxicology. 2005;215(3):227-33. doi: 10.1016/j.tox.2005.07.009.

24. Nishino $Y$, Takemura S, Minamiyama $Y$, Hirohashi K, Ogino $\mathrm{T}$, Inoue $\mathrm{M}$, et al. Targeting superoxide dismutase to renal proximal tubule cells attenuates vancomycin-induced nephrotoxicity in rats. Free Radic Res. 2003;37(4):373-9. doi: 10.1080/1071576031000061002

25. Vasco CF, Watanabe M, Fonseca CDD, Vattimo MFF. Sepsis- 
induced acute kidney injury: kidney protection effects by antioxidants. Rev Bras Enferm. 2018;71(4):1921-7. doi: 10.1590/0034-7167-2017-0469.

26. Barreiro E, Sánchez D, Gáldiz JB, Hussain SN, Gea J. ENIGMA in COPD project. N-acetylcysteine increases manganese superoxide dismutase activity in septic rat diaphragms. Eur Respir J. 2005;26(6):1032-9. doi: 10.1183/09031936.05.00003705.

27. Villagrasa V, Cortijo J, Martı'-Cabrera M, Ortiz JL, Berto L, Esteras $\mathrm{A}$, et al. Inhibitory effects of $\mathrm{N}$-acetylcysteine on superoxide anion generation in human polymorphonuclear leukocytes. J Pharm Pharmacol. 1997;49(5):525-9. 10.1111/ j.2042-7158.1997.tb06836.x.
28. Victor VM, Rocha M, de la Fuente M. N-acetylcysteine protects mice from lethal endotoxemia by regulating the redox state of immune cells. Free Radic Res. 2003;37(9):919-29. doi: 10.1080/1071576031000148727.

29. Mehta R, Kellum J, Shah S, Molitoris B, Ronco C, Warnock DG, et al. Acute Kidney Injury Network. Report of an initiative to improve outcomes in acute kidney injury. Crit Care. 2007;11(2):R31. doi: 10.1186/cc5713.

30. Coca S, Yalavarthy R, Concato J, Parikh C. Biomarkers for the diagnosis and risk stratification of acute kidney injury: a systematic review. Kidney Int. 2008;73(9):1008-16. doi: 10.1038/sj.ki.5002729. 